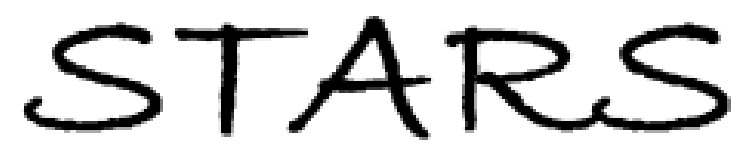

University of Central Florida

STARS

$1-1-2010$

\title{
Bound on quantum computation time: Quantum error correction in a critical environment
}

\author{
E. Novais
}

Eduardo R. Mucciolo

University of Central Florida

Harold U. Baranger

Find similar works at: https://stars.library.ucf.edu/facultybib2010

University of Central Florida Libraries http://library.ucf.edu

This Article is brought to you for free and open access by the Faculty Bibliography at STARS. It has been accepted for inclusion in Faculty Bibliography 2010 s by an authorized administrator of STARS. For more information, please contactSTARS@ucf.edu.

\section{Recommended Citation}

Novais, E.; Mucciolo, Eduardo R.; and Baranger, Harold U., "Bound on quantum computation time: Quantum error correction in a critical environment" (2010). Faculty Bibliography 2010s. 599. https://stars.library.ucf.edu/facultybib2010/599

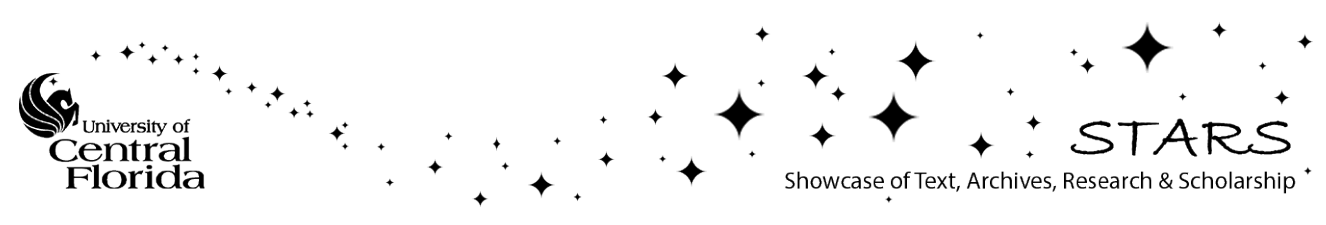




\title{
Bound on quantum computation time: Quantum error correction in a critical environment
}

\author{
E. Novais, ${ }^{1}$ Eduardo R. Mucciolo, ${ }^{2}$ and Harold U. Baranger ${ }^{3}$ \\ ${ }^{1}$ Centro de Ciências Naturais e Humanas, Universidade Federal do ABC, Santo André, São Paulo, Brazil \\ ${ }^{2}$ Department of Physics, University of Central Florida, Box 162385, Orlando, Florida 32816, USA \\ ${ }^{3}$ Department of Physics, Duke University, Box 90305, Durham, North Carolina 27708-0305, USA
}

(Received 20 April 2010; published 31 August 2010)

\begin{abstract}
We obtain an upper bound on the time available for quantum computation for a given quantum computer and decohering environment with quantum error correction implemented. First, we derive an explicit quantum evolution operator for the logical qubits and show that it has the same form as that for the physical qubits but with a reduced coupling strength to the environment. Using this evolution operator, we find the trace distance between the real and ideal states of the logical qubits in two cases. For a super-Ohmic bath, the trace distance saturates, while for Ohmic or sub-Ohmic baths, there is a finite time before the trace distance exceeds a value set by the user.
\end{abstract}

DOI: 10.1103/PhysRevA.82.020303

PACS number(s): 03.67.Pp, 03.65.Yz, 03.67.Ac

\section{INTRODUCTION}

All physical implementations of quantum information processing face the inexorable reality of being embedded in an environment that causes decoherence [1]. There are many strategies for dealing with this fact [2], quantum error correction (QEC) being one of the most versatile [3]. As QEC will likely be used in any large-scale implementation of quantum information processing, it is important to define and to quantify its limits.

There have been several recent advances in understanding the limits of fault tolerant quantum computing [4]. Part of this effort has been on proving resilience, the notion that any desired accuracy of quantum computation may be attained by concatenating levels of the QEC code [5]. In particular, arguments for resilience in correlated environments have been constructed either by using techniques based on sums over faulty paths $[6,7]$ or by reducing the problem to an almost stochastic one through scaling [8]. In this Rapid Communication, we focus on a related question: Given a certain quantum computer and an environment, what is an upper bound on the time available for computation? We provide an answer for a broad class of environments using a Hamiltonian formulation, including those where correlation effects are induced by gapless modes (i.e., critical environments).

The main results of our argument are as follows. First, the coarse-grained quantum evolution of logical qubits is essentially the same as that of physical qubits, up to a renormalized coupling to the environment. Thus, QEC maps our generic environment-computer interaction model onto itself, a property, which has proven useful for other ways of suppressing decoherence, such as dynamical decoupling [9]. Second, we use this coarse-grained quantum evolution to find the maximum time available for computation, as assessed by the trace distance between the real and ideal states of the computer. There is a regime where computation can continue indefinitely and so is resilient, while in other regimes, the maximum time depends strongly on the QEC code, number of logical qubits, and environment-computer interaction.

\section{ENVIRONMENT-COMPUTER INTERACTION AND HYPOTHESES}

Many physical noise cases can be directly modeled by the spin-boson model [10]. However, as originally proposed [11], this model has a much more fundamental appeal.

Let us assume that the qubits are already under the protection of an initial strategy, such as a decoherence-free subspace or dynamical decoupling [12]. Though it deals with the dominant effect, it is unlikely to solve the decoherence problem completely. The inevitable residual interaction between the computer and the environment imposes a pointer basis for the qubits, which we call the $z$ direction of each individual qubit. Another reasonable assumption is that the environment consists of a very large set of quantum degrees of freedom with some intrinsic dissipative mechanism. Hence, in the absence of the qubits, the environment will be in a local minimum of its energy landscape. Our next (crucial) assumption is that linear response describes the influence of the computer on the environment. In that case, the computer-environment interaction cannot take the environment out of its local minimum, and so we may use the harmonic approximation to describe the quantum fluctuations of the environmental degrees of freedom. By construction, then, the environment-computer interaction term is linear. Thus, we use the well-known and experimentally relevant spin-boson model to discuss the limits on protection that QEC and fault-tolerant methods can yield.

Having thus settled the model of the strongest channel of residual decoherence, we consider the presence of an additional weaker transverse channel, denoted by $x$. If the first channel were absent, the preceding argument applied to the transverse channel leads to a model of the same form but with a much weaker coupling: $\lambda_{x} \ll \lambda_{z}$. Hence, we describe the residual decoherence of the quantum computer by two bosonic baths $(\hbar=1)$,

$$
H_{0}=\sum_{\alpha=\{x, z\}} \sum_{|\mathbf{k}| \neq 0} \omega_{\alpha, k} a_{\alpha, \mathbf{k}}^{\dagger} a_{\alpha, \mathbf{k}} .
$$

The $a_{\alpha, \mathbf{k}}$ obey standard commutation relations, and $\omega_{\alpha, k}=$ $\omega_{0}\left(|\mathbf{k}| / k_{0}\right)^{z_{\alpha}}$, where $k_{0}$ and $\omega_{0}$ are constants with dimensions of momentum and frequency, respectively, and $z_{\alpha}$ is a 
dynamical exponent. The environment-computer interaction has the form

$$
H_{I}=\sum_{\alpha=\{x, z\}} \sum_{\mathbf{x}} \lambda_{\alpha}: f^{\alpha}(\mathbf{x}): \sigma_{\mathbf{x}}^{\alpha}
$$

where $\sigma_{\mathbf{x}}^{x, z}$ are the Pauli matrices for the qubit at position $\mathbf{x},::$ stands for normal ordering, and $: f^{\alpha}(\mathbf{x}):=(2 \pi / L)^{D / 2}$ $\sum_{\mathbf{k} \neq 0}\left(u_{\alpha, k} e^{i \mathbf{k} \cdot \mathbf{x}} a_{\alpha, \mathbf{k}}^{\dagger}+\right.$ H.c. $)$, with $\left|u_{\alpha, k}\right|^{2}=\kappa_{0}^{-D}\left(|\mathbf{k}| / k_{0}\right)^{2 s_{\alpha}}$, which defines the exponent $s_{\alpha}$. Here, the environment has spatial dimension $D$ and smallest momentum $2 \pi / L$, and $\kappa_{0}$ is a constant with dimensions of momentum. There is no a priori restriction on including a third bath; however, it would be a redundant description of the possible errors. All the bosonic averages performed are done with respect to the bosonic vacuum with no initial entanglement between the computer and the bath. If there were some initial entanglement, it could be modeled using a finite temperature in the bosonic correlators, thus introducing an exponential scale. Since our goal is to calculate an upper bound for the computational time, we do not consider this case.

In conjunction with this model, we make some assumptions about the computer and the QEC method. (i) Gates are flawless and are done much faster than the QEC period $\Delta$. (ii) State preparation and measurements are done perfectly. (iii) Lowest-order perturbation theory in $H_{I}$ is enough to describe the evolution during a QEC step. (iv) All the syndromes indicate a nonerror result; that is, we consider the most favorable quantum computer evolution, as any other evolution will involve a larger leak of information to the environment [8].

\section{UNCORRECTABLE ERRORS AND THE QUANTUM EVOLUTION}

The first step of the quantum calculation is to consider the evolution operator in the interaction picture $U_{I}(\Delta, 0)=$ $T_{t} \exp \left[-i \int_{0}^{\Delta} d t H_{I}(t)\right]$ up to a time $\Delta^{-}$, just before error correction is applied. QEC divides errors into classes that can be distinguished from each other; however, within each class, different errors are not differentiated by the syndrome. For each logical qubit, the syndrome breaks the evolution into $u\left(\Delta^{+}, 0\right)=\sum_{i=0}^{N-1} v_{i}$, where $i$ indexes the $N$ possible syndromes of that qubit [8]. Each one of these evolution operators has a good and a bad part: $v_{i}=\alpha_{i}+\sum_{j=1}^{3} \beta_{i}^{j} \bar{\sigma}^{j}$, where $\bar{\sigma}^{j}$ represents a logical error. These logical errors are uncorrectable (or bad evolutions [13]).

Following our hypotheses, within the QEC period $\Delta$, we approximate the evolution operator by expanding to lowest order in the couplings $\lambda_{\alpha}$. (Technically, the expansion parameters are $\lambda_{\alpha} \Delta$ and not simply $\lambda_{\alpha}$.) Thus, for a single qubit,

$$
U_{I}(\Delta, 0) \approx 1-i \sum_{\alpha=\{x, z\}} \sum_{\mathbf{x}} \lambda_{\alpha} \Delta: f^{\alpha}(\mathbf{x}, 0): \sigma_{\mathbf{x}}^{\alpha} .
$$

For a code of distance $d_{c}$, one finds that the lowest-order term that must be kept in each logical qubit is of order $d_{c}$ in the coupling to the environment. For illustration, consider the smallest distance-3 code, namely, the 5-qubit code [2]. At the end of a QEC period, there are $4^{5}$ possible configurations for the five qubits. They are divided into $4^{2}$ groups with distinct syndromes; however, each group has $4^{3}$ elements that cannot be distinguished by the code. We choose to analyze the evolution for which all the syndromes are the no-error type. This yields the quantum evolution operator,

$$
\begin{aligned}
v_{0}(\Delta, 0) \approx & \overline{1}+i \Delta^{3} \sum_{\mathbf{x}, \alpha, \beta, i, j, k} \eta_{i j k}^{\alpha \beta} \lambda_{\alpha} \lambda_{\beta}^{2} \\
& \times: f^{\alpha}\left(\mathbf{x}_{i}, 0\right):: f^{\beta}\left(\mathbf{x}_{j}, 0\right):: f^{\beta}\left(\mathbf{x}_{k}, 0\right): \bar{\sigma}_{\mathbf{x}}^{\alpha},
\end{aligned}
$$

with $\mathbf{x}$ labeling the logical qubits, $\alpha, \beta=\{x, z\}$, and $i, j, k=\{1, \ldots, 5\}$ labeling the physical qubits inside the logical qubit $\mathbf{x}$. Each coefficient $\eta_{i j k}^{\alpha \beta}$ has two possible values $\eta_{324}^{x z}=\eta_{435}^{x z}=\eta_{514}^{x z}=\eta_{125}^{x z}=\eta_{213}^{x z}=\eta_{134}^{z x}=\eta_{412}^{z x}=$ $\eta_{245}^{z x}=\eta_{523}^{z x}=\eta_{315}^{z x}=1$ and zero for all other indices.

Now, we use the commutation relations of the free bosons to normal order the evolution operator in Eq. (4),

$$
\begin{aligned}
v_{0}(\Delta, 0) \approx & \overline{1}+i \sum_{\mathbf{x}, \alpha, \beta, i, j, k} \eta_{i j k}^{\alpha \beta} \lambda_{\alpha} \Delta: f^{\alpha}\left(\mathbf{x}_{i}, 0\right): \\
& \times\left[a_{\beta j k}+\left(\lambda_{\beta} \Delta\right)^{2}: f^{\beta}\left(\mathbf{x}_{j}, 0\right) f^{\beta}\left(\mathbf{x}_{k}, 0\right):\right] \bar{\sigma}_{\mathbf{x}}^{\alpha},
\end{aligned}
$$

where $\quad a_{\alpha i j}=\left(\lambda_{\alpha} \Delta\right)^{2} \sum_{\mathbf{k} \neq 0}\left|u_{\alpha, k}\right|^{2} \exp \left[-i \mathbf{k} \cdot\left(\mathbf{x}_{i}-\mathbf{x}_{j}\right)\right]$. Equation (5) is written for the 5-qubit code with no concatenation; it is straightforward to generalize it to a larger distance or concatenated code. For instance, the level-1 concatenated code requires 25 physical qubits with the coefficients $\eta$ changing accordingly. In this case, $\Delta$ includes the time needed to extract all syndromes (including level-1 syndromes), and uncorrectable errors appear at higher order in $\lambda_{\alpha}$.

The evolution operator for a logical qubit can be abridged by rewriting Eq. (5) as

$$
v_{0}(\Delta, 0) \approx \overline{1}+i \Delta \sum_{\mathbf{x}, \alpha=\{x, z\}}\left(\lambda_{\alpha}^{*}+\Gamma_{\alpha}\right): f^{\alpha}(\mathbf{x}, 0): \bar{\sigma}_{\mathbf{x}}^{\alpha},
$$

where $\mathbf{x}$ is the average position of the physical qubits belonging to the logical qubit, $\lambda_{\alpha}^{*} \equiv \lambda_{\alpha} \sum_{\beta, i, j, k} \eta_{i j k}^{\alpha \beta} a_{\beta j k}$ is the effective coupling constant, and $\Gamma_{\alpha}(\mathbf{x}, 0) \equiv \lambda_{\alpha} \sum_{\beta, i, j, k} \eta_{i j k}^{\alpha \beta}\left(\lambda_{\beta} \Delta\right)^{2}$ $: f^{\beta}\left(\mathbf{x}_{j}, 0\right) f^{\beta}\left(\mathbf{x}_{k}, 0\right)$ : accounts for higher-order corrections. The latter dresses the single logical qubit amplitude $a_{\beta j k}$ by the interactions with other logical qubits. If the spatial separation of logical qubits is at least $\Xi$ while that of the physical qubits within a logical qubit is $\xi$, then $\Gamma_{\alpha}$ generates corrections of order $(\xi / \Xi)^{4 \delta_{\alpha}}$ to observable quantities, where $\delta_{\alpha}$ is the smallest scaling dimension of the $f_{\alpha}$. For simplicity, we assume that $\xi \ll \Xi$; hence, since we are seeking an upper bound on the computing time, we can ignore the $\Gamma_{\alpha}$ corrections.

Another scenario to consider is when the physical qubits do not interact with each other $\xi \rightarrow \infty$. In this case, $a_{\beta j k} \rightarrow 0$ and the leading correction will come from $\Gamma_{\alpha}$. This demands a slightly different organization of the argument: It leads to a different definition of the effective coupling constant but does not imply that there are no uncorrectable errors (see, e.g., Ref. [13] for the stochastic error model). Most of the following discussion can be readily adapted to this case following arguments similar to those in Ref. [8], which we therefore omit here. 
The steps outlined earlier result in the following quantum evolution operator for the logical qubits:

$$
\bar{U}_{I}(T, 0) \approx T_{t} e^{i \int_{0}^{T} d t \sum_{\mathbf{x}, \alpha=\{x, z\}} \lambda_{\alpha}^{*}: f^{\alpha}(\mathbf{x}, t): \bar{\sigma}_{\mathbf{x}}^{\alpha}} .
$$

As a direct consequence of the coarse graining used in Eq. (3), note that the ultraviolet frequency cutoff is $\Delta^{-1}$.

Equation (7) is a remarkable expression: It shows that in the long wavelength limit, the logical qubits obey the same dynamics as the physical qubits. In other words, QEC maps the spin-boson decoherence model onto itself. There are, of course, several ways to reduce $\lambda_{\alpha}^{*}$ : (i) engineer the position of the physical qubits, (ii) change the distance of the code, or (iii) concatenate the code. Nevertheless, as long as $\lambda_{\alpha}^{*}$ and $\Gamma_{\alpha}$ are not strictly zero, there will be degradation of the information in the logical qubits. Thus, Eq. (7) implicitly defines the largest time scale potentially available for computing.

\section{UPPER-BOUND ON THE COMPUTATIONAL TIME}

One way to quantify the loss of quantum information to the environment is through the trace distance [2] between the reduced density matrix $\rho_{R}(T)$ and the ideal density matrix $\rho_{0}: D\left(\rho_{R}(T), \rho_{0}\right)=\frac{1}{2} \operatorname{tr}\left|\rho_{R}(T)-\rho_{0}\right|$. The trace distance indicates how hard it is to distinguish two density matrices by performing measurements; hence, it is a natural way to quantify how well QEC protects information. Let us suppose that there is a criterion $D\left(\rho_{R}(T), \rho_{0}\right) \leqslant D_{\text {crit }}$ for a successful computation. Our goal, then, is to evaluate the time $T$ available for computation.

Since we expect that $D\left(\rho_{R}(T), \rho_{0}\right)$ is small, it is natural to formulate the problem in powers of the effective couplings $\lambda_{\alpha}^{*}$. For an upper bound on $T$, we can stop the perturbative expansion in second order. Though it is difficult to evaluate $D\left(\rho_{R}(T), \rho_{0}\right)$, in general, we can make some progress by considering two distinct cases. First, we look at an isolated logical qubit, namely, $\Xi \rightarrow \infty$. Second, we use the HilbertSchmidt norm to bound the trace distance and to define an upper bound on $T$ in general.

\section{INFORMATION LOST BY A SINGLE LOGICAL QUBIT}

For a single logical qubit, the trace distance can be expressed in terms of the expectation values of the logical qubit $D\left(\rho_{R}(T), \rho_{0}\right)=\sqrt{\left|\delta \sigma^{+}(T)\right|^{2}+\left[\delta \sigma^{z}(T)\right]^{2} / 4}$, where $\delta \sigma^{\alpha}(T)=\left\langle\bar{\sigma}^{\alpha}(T)\right\rangle-\left\langle\bar{\sigma}^{\alpha}\right\rangle$ and, for convenience, we dropped the space label. Since the largest coupling constant is in the $z$ direction, we employ a rotation to take it into account nonperturbatively. First, we define the operator: $F^{z}[(n+1) \Delta]:-$ $: F^{z}(n \Delta):=\lambda_{z}^{*} \Delta: f^{z}(n \Delta):$ and then rotate the evolution operator at each $n$th QEC period using the unitary transformation $e^{-i: F^{z}(n \Delta): \bar{\sigma}^{z}}$. This rotation cancels the $z$ component of $H_{I}$ at the expense of dressing the transverse coupling. The rotated interacting Hamiltonian at a time $t=n \Delta$ can be written as $H_{I}^{\mathrm{rot}}(t)=\lambda_{x}^{*} \sum_{\alpha=\{ \pm\}}: f^{\alpha}(t): \exp \left[-2 i \alpha: F^{z}(t):\right] \bar{\sigma}^{\alpha}$.

We can now calculate the expectation values $\delta \sigma^{\alpha}(T)$ in perturbation theory in $\lambda_{x}^{*}$. This is a simple but tedious calculation, which we omit here. For our purposes, the main feature appears already at zeroth order (dephasing only). In this case, it is straightforward to show that $\left\langle\bar{\sigma}^{z}(T)\right\rangle=\left\langle\bar{\sigma}^{z}\right\rangle$ and $\left\langle\bar{\sigma}^{+}(T)\right\rangle=$ $e^{-4 \gamma_{z}(T)}\left\langle\bar{\sigma}^{+}\right\rangle$, where $\gamma_{z}(T)=(2 \pi / L)^{D}\left(\lambda_{z}^{*}\right)^{2} \sum_{\mathbf{k} \neq 0} \frac{\left|u_{z, k}\right|^{2}}{\omega_{z, k}^{2}}[1-$ $\left.\cos \left(\omega_{z, k} T\right)\right]$ is the well-known decoherence function [14]. We thus obtain

$$
D\left(\rho_{R}(T), \rho_{0}\right)=\left|\left\langle\bar{\sigma}^{+}\right\rangle\right|\left[1-e^{-4 \gamma_{z}}(T)\right]
$$

By defining $\zeta_{z}=2\left(z_{z}-s_{z}\right)-D$, we can distinguish the following decoherence regimes in the long-time limit:

$$
\gamma_{z}(M \Delta) \propto \begin{cases}\left(\lambda_{z}^{*} / \omega_{0}\right)^{2}\left(\omega_{0} \Delta\right)^{-\zeta_{z} / z_{z}}, & \zeta_{z}<0, \\ \left(\lambda_{z}^{*} / \omega_{0}\right)^{2} \ln M, & \zeta_{z}=0, \\ \left(\lambda_{z}^{*} / \omega_{0}\right)^{2}\left(\omega_{0} \Delta\right)^{\zeta_{z} / z_{z}} M^{\zeta_{z} / z_{z}}, & 0<\zeta_{z}<2 z_{z}, \\ \left(\lambda_{z}^{*} \Delta\right)^{2}\left(k_{0} L / 2 \pi\right)^{\zeta_{z}-2 z_{z}} M^{2}, & \zeta_{z}>2 z_{z},\end{cases}
$$

where $M \equiv T / \Delta$ is the number of QEC steps that are performed. These regimes are straightforward generalizations of the super-Ohmic $\left(\zeta_{z}<0\right)$, Ohmic $\left(\zeta_{z}=0\right)$, and sub-Ohmic $\left(\zeta_{z}>0\right)$ regimes. Notice that for $\zeta_{z}<0$, the trace distance will converge to a finite value $D_{\text {sat }}$. Equation (8) is an exact result, but we expect $D_{\text {crit }}$ to be small. Hence, we can expand the exponential and find the maximum time for computation with isolated logical qubits. Assuming $D_{\text {crit }}>D_{\text {sat }}$, we find

$$
M_{\max } \propto \begin{cases}\infty, & \zeta_{z}<0, \\ \exp \left[c_{D, z} D_{\text {crit }}\left(\omega_{0} / \lambda_{z}^{*}\right)^{2}\right], & \zeta_{z}=0, \\ D_{\text {crit }}^{z_{z} / \zeta_{z}}\left(\omega_{0} / \lambda_{z}^{*}\right)^{2 z_{z} / \zeta_{z}} /\left(\omega_{0} \Delta\right), & 0<\zeta_{z}<2 z_{z}, \\ \left(2 \pi / k_{0} L\right)^{\left(\zeta_{z}-2 z_{z}\right)} \sqrt{D_{\text {crit }}} /\left(\lambda_{z}^{*} \Delta\right), & \zeta_{z}>2 z_{z},\end{cases}
$$

where $c_{D, z}$ is a dimensionless prefactor of order unit.

\section{UPPER BOUND FOR MULTIPLE LOGICAL QUBITS}

To find an upper bound on the trace distance when logical qubits are not isolated, we use the subadditivity property of the square-root function and an inequality proved in Ref. [15],

$$
D_{\mathrm{HS}}\left(\rho_{R}(T), \rho_{0}\right) \leqslant D\left(\rho_{R}(T), \rho_{0}\right) \leqslant 2^{N / 2} D_{\mathrm{HS}}\left(\rho_{R}(T), \rho_{0}\right),
$$

where $D_{\mathrm{HS}}\left(\rho_{R}(T), \rho_{0}\right)=\frac{1}{2}\left[\operatorname{tr}\left|\rho_{R}(T)-\rho_{0}\right|^{2}\right]^{1 / 2}$ is the HilbertSchmidt norm and $N$ is the number of logical qubits. Following a similar procedure to that used for the trace distance, we can expand $D_{\mathrm{HS}}\left(\rho_{R}(T), \rho_{0}\right)$ to second order in $\lambda_{\alpha}^{*}$,

$$
\begin{array}{r}
D_{\mathrm{HS}}\left(\rho_{R}(T), \rho_{0}\right) \propto \sqrt{\sum_{\alpha}\left(\lambda_{\alpha}^{*}\right)^{2}\left|\sum_{\mathbf{x}, \mathbf{y}} W_{\mathbf{x}, \mathbf{y}}^{\alpha}(T)\right|^{2}}, \\
W_{\mathbf{x}, \mathbf{y}}^{\alpha}(T)=\left(\frac{2 \pi}{L}\right) \sum_{\mathbf{k} \neq 0} \frac{\left|u_{\alpha, k}\right|^{2}}{\omega_{\alpha, k}^{2}} e^{-i \mathbf{k} \cdot(\mathbf{x}-\mathbf{y})}\left(1-e^{-i \omega_{\alpha, k} T}\right) .
\end{array}
$$

There are two types of $W_{\mathbf{x}, \mathbf{y}}^{\alpha}(T)$ : (i) the diagonal self-interaction terms, and (ii) the correlation terms in which pairs of 
logical qubits interact. Both types lead to the same functional dependence in the sum:

$$
\left|\sum_{\mathbf{x}, \mathbf{y}} W_{\mathbf{x}, \mathbf{y}}^{\alpha}(T)\right| \propto \begin{cases}N \omega_{0}^{-1}\left(\omega_{0} \Delta\right)^{-\zeta_{\alpha} / z_{\alpha}}, & \zeta_{\alpha}<0, \\ N \omega_{0}^{-1} \ln M, & \zeta_{\alpha}=0, \\ N \omega_{0}^{-1}\left(\omega_{0} \Delta M\right)^{\zeta_{\alpha} / z_{\alpha}}, & 0<\zeta_{\alpha}<z_{\alpha}, \\ N \Delta\left(k_{0} L / 2 \pi\right)^{\zeta_{\alpha}-z_{\alpha}} M, & \zeta_{\alpha}>z_{\alpha},\end{cases}
$$

where the proportionality constant is of order 1 . However, the two types of terms lead to different onset criteria. For the self-interacting part, the different regimes are delineated using $\zeta_{\alpha}=2\left(z_{\alpha}-s_{\alpha}\right)-D$, while for the correlation part, the spatial sum leads to $\zeta_{\alpha}=2\left(z_{\alpha}-s_{\alpha}\right)+D_{x}-D$ with $D_{x}$ being the dimension of the qubit array. Note that some physical arrangements of qubits are more favorable than others; for instance, a linear architecture is more favorable than a square or cubic one.

First, note that in order to apply QEC, we assumed $\lambda^{* 2} N \ll$ 1 [Eqs. (12) and (14)]. Second, for a given critical distance $D_{\text {crit }}$ and using Eq. (14), we arrive at an upper bound on the time available to compute due to each component of the environment:

$$
M_{\max }= \begin{cases}\infty, & \zeta_{\alpha}<0, \\ \exp \left[\frac{b_{D, \alpha} D_{\text {crit }}}{N\left(\lambda_{z}^{*} / \omega_{0}\right)}\right], & \zeta_{\alpha}=0, \\ \left(\omega_{0} \Delta\right)^{-1}\left[\frac{D_{\text {crit }}}{N\left(\lambda_{z}^{*} / \omega_{0}\right)}\right]^{z_{\alpha} / \zeta_{\alpha}}, & 0<\zeta_{\alpha}<z_{\alpha}, \\ \left(2 \pi / k_{0} L\right)^{\zeta_{\alpha}-z_{\alpha}} \frac{D_{\text {crit }}}{N\left(\lambda_{z}^{*} \Delta\right)}, & \zeta_{\alpha}>z_{\alpha},\end{cases}
$$

where $b_{D, \alpha}$ is a dimensionless constant. If $\lambda_{x} \sim \lambda_{z} \sim \lambda$, this result is simply related to the code distance or the level of concatenation: $\lambda_{\alpha}^{*} \sim \lambda^{d_{c}}$.

\section{CONCLUSIONS}

For how long is it possible to quantum compute? (1) The trace distance calculations give us a rule of thumb: For a finite computation time, the residual decoherence of a logical qubit after the first QEC step times the number of logical qubits must be a small number, $\left(\lambda_{\alpha}^{*}\right)^{2} N \ll 1$. (The case of no spatial correlation among the physical qubits at short times $\lambda_{\alpha}^{*}=0$ was discussed in Ref. [8].) This condition must be a factor in the choice of the distance of the code or concatenation level. (2) While the argument presented here does not directly address the threshold theorem, the upper bound on the available computational time shows that there are certain limits to the power of QEC. The three regimes that we find nicely fit the qualitative interpretation of resilience as a dynamical quantum phase transition [8]. (2.1) For $\zeta_{x, z}<0$ (above the upper critical dimension), the usual enunciation of the threshold theorem [6] can be used, and therefore it is possible to compute indefinitely. (2.2) For $\zeta_{x, y}>z_{x, y}$ (below the lower critical dimension), correlations are so strong that the available computational time is formally zero (since it depends on the size of the bath $L$ ). It is, however, conceivable that its strong infrared divergence may be handled by combining dynamical decoupling and QEC methods [9]. (2.3) Finally, between these two regions, there is a maximum time available to compute. This constraint must also be a factor in the choice of the distance of the code or concatenation level. Even though the regimes fit into the general discussion of Ref. [8], the definition of the upper critical dimension given here is not the same. The reason is that, we have now shown that it is possible to explicitly treat a dense set of qubits.

\section{ACKNOWLEDGMENTS}

We thank D. Lidar for useful discussions and correspondence. This work was partially supported by the Office of Naval Research and CNPq-Brazil.
[1] P. C. E. Stamp and A. Gaita-Arino, J. Mater. Chem. 19, 1718 (2009).

[2] M. A. Nielsen and I. L. Chuang, Quantum Computation and Quantum Information (Cambridge University Press, Cambridge, UK, 2000).

[3] A. M. Steane, Phys. Rev. Lett. 77, 793 (1996); A. R. Calderbank and P. W. Shor, Phys. Rev. A 54, 1098 (1996); E. Knill and R. Laflamme, ibid. 55, 900 (1997); D. Gottesman, Phys. Rev. A 57, 127 (1998).

[4] Quantum Error Correction, edited by D. Lidar, T. Brun, and P. Zanardi (unpublished); M. Plenio and S. Virmani, New J. Phys. 12, 033012 (2010); J. Kempe et al., Lecture Notes in Computer Science (Springer, Berlin, 2010), Vol. 5125, p. 845.

[5] E. Knill, R. Laflamme, and W. H. Zurek, Science 279, 342 (1998); D. Aharonov and M. Ben-Or, e-print arXiv:quant-ph/9906129.

[6] B. M. Terhal and G. Burkard, Phys. Rev. A 71, 012336 (2005); P. Aliferis, D. Gottesman, and J. Preskill, Quantum Inf. Comput. 6, 97 (2006); D. Aharonov, A. Kitaev, and J. Preskill, Phys. Rev. Lett. 96, 050504 (2006).
[7] H. K. Ng and J. Preskill, Phys. Rev. A 79, 032318 (2009).

[8] E. Novais and H. U. Baranger, Phys. Rev. Lett. 97, 040501 (2006); E. Novais, E. R. Mucciolo, and H. U. Baranger, ibid. 98, 040501 (2007); Phys. Rev. A 78, 012314 (2008).

[9] H. K. Ng, D. A. Lidar, and J. Preskill, e-print arXiv:0911.3202.

[10] Y. Makhlin, G. Schön, and A. Shnirman, Rev. Mod. Phys. 73, 357 (2001); L. Chirolli and G. Burkard, Adv. Phys. 57, 225 (2008).

[11] A. O. Caldeira and A. J. Leggett, Ann. Phys. 149, 374 (1983); A. J. Leggett et al., Rev. Mod. Phys. 59, 1 (1987).

[12] D. A. Lidar, I. L. Chuang, and K. B. Whaley, Phys. Rev. Lett. 81, 2594 (1998); L. Viola, E. Knill, and S. Lloyd, ibid. 82, 2417 (1999).

[13] [http://www.theory.caltech.edu/people/preskill/ph229/notes/ chap7.pdf].

[14] H.-P. Breuer and F. Petruccione, The Theory of Open Quantum Systems (Oxford University Press, Oxford, 2002).

[15] S. Popescu, A. J. Short, and A. Winter, Nat. Phys. 2, 754 (2006). 